\title{
Consideration on the Power System Planning in the Urbanized Construction of Yunnan's Mountainous Areas
}

\author{
Yunhong GONG \\ Qujing Normal University, \\ Qujing, Yunnan Province, 655011 China
}

\begin{abstract}
Constructing Urban Areas in Mountains" is one of the important measures to realize the goal of comprehensively constructing a well-off society in Yunnan. All the projects to be constructed in mountainous areas can't depart from the support of electric power, so giving top priority to electric power has become the first precondition for building urban areas in mountains in Yunnan. On the basis of analyzing the main problems in the power system planning in the construction of the urban areas in Yunnan's mountains, in this paper, several strategies for the power system planning in the construction of the urban areas in Yunnan's mountains are optimized from enhancing the communication between power supply enterprises and government, including the power grid planning into the overall planning of the urbanized construction of mountainous areas, making the load prediction of power system planning based on the construction plan of mountainous area urbanization, adhering to the actual local conditions in the planning objective of power distribution network, advocating appropriate advances and insisting on the sustainable development strategy, and co-constructing a resource conservation-minded society.
\end{abstract}

Keywords: Constructing Urban Areas in Mountains; Mountainous Area Urbanization; Power System Planning

\section{INTRODUCTION}

The report of the CPC's Eighteenth Congress clearly showed, "Urbanization is a historical mission in the modernized construction of China and closely intertwines with the agricultural modernization; the healthy development of urbanization is necessarily promoted by abiding by the objective law of urbanization". In recent 10 years, the progress of the urbanization has been obviously accelerated. The report of the CPC's Eighteenth Congress also presented, "it is urgent to promote the integration of urban and rural development" and "the integration of urban and rural development is the fundamental way to solve the problems of agriculture, rural areas and peasants". For Yunnan province in which frontier minority areas are concentrated the task of the urbanization is much heavier because of the impact from many factors such as geography and culture.

Since the implementation of China's reform and opening-up policy, tremendous achievements have been made by Yunnan province in the economic and social development, and the urbanization rate has increased from $12.8 \%$ to $12.8 \%$. Therefore, Yunnan province has entered the stage of the accelerated development of urbanization, but remains to be far away from the national average.

After 30 years of China's reform and opening-up policy, an urbanization path with special Chinese characteristic has been explored for China's urbanization. The typical urbanization models include "Southern Jiangsu Model", "Wenzhou Model", "Xiangjiang Model", etc. On the whole, the urbanization rate of the eastern developed areas has been very harmonious with their economic development level, but the urbanization development in frontier minority areas remains to be slow. In Yunnan, mountain and semi-mountainous areas 
account for $94 \%$ of the total area of the province, and flatlands (basin and river valley) are only $6 \%$. Under the strategy of speeding up the construction of southwest China's "bridgehead" opening up to the outside world, how to promote the urbanization and crack the contradictions between the urban land use and agricultural cultivated land protection in the economic and social development has been a very important issue necessary for Yunnan to deal with in the future. On September 5, 2011, Yunnan's conference about the protection of flatland farmland in the construction of mountainous areas was held in Dali, on which Guangrong QIN, Yunnan's provincial party committee secretary, stressed, "The construction is necessarily based on the actual conditions of Yunnan province; every effort is necessarily made to realizing the goal of efficient land utilization and scientific urbanized development according to the general requirements of protecting red culture, making an overall plan for the urban and rural development, constructing urban areas in mountains, and promoting peasants to work in urban areas, so that a urbanization path with the special characteristics of Yunnan can be explored.

The report of the Economic research institute of Yunnan province shows the general idea for constructing urban areas in mountains - a Slope Layered Gradient Development Model. According to the blueprint, Yunnan is supposed to gain a harmonious situation in 4 years, in which landscapes and countryside form beautiful sceneries, urban and rural areas are integrated, urban areas develop along mountain slopes, and farmland remains to be available for offspring to cultivate.

Therefore, it is seen that "constructing urban areas in mountains and promoting peasants to work in urban areas" is one of the important measures to realize the goal of comprehensively constructing a well-off society in Yunnan. All projects to be constructed in mountainous areas, including constructing urban areas, industrial parks and scenic spots in mountains, can't depart from the support of electric power, so giving top priority to electric power has become the first precondition for building urban areas in mountains in Yunnan. Electric power industry is supposed to make due contributions to promoting the project of "constructing urban areas in mountains" in Yunnan.

\section{The main problem in the power system planning for the urbanization of Yunnan's mountainous areas}

Power system planning is to determine when and where to set up what type of circuits or transformers for meeting the requirements for the future load growth and power supply development. The pace of the urbanization of Yunnan's mountainous areas is being accelerated, and power system planning is imminent. Concluding the past experience, the main problem in the power system planning for the urbanization of Yunnan's mountainous areas is that the coordination and cohesion between power system planning and the overall planning of the urbanization of Yunnan's mountainous areas remain to be further strengthened. The specifics can be reflected from the following aspects:

\section{1 "Plan" often can't catch up with "change" in the process of power system construction}

With the implementation of the project of "constructing urban areas in mountains", the power system planning progress needs to be accelerated. In the process of power system planning, the selection of substation sites and lines will often encounter difficulties. Specifically, substation sites and lines can't be determined even if government communicates with the local people repeatedly; the substation sites and lines, which have been determined, often change many times in the process of construction. 


\subsection{Unscientific power system planning}

Power system planning is often compiled by power grid enterprises independently and cannot fully integrate with the local economic and social development trend and urban development because of the information asymmetry. The goal of some plans is too high or too low and becomes unscientific, so that it is greatly different from the planning and construction of the urbanization of Yunnan's mountainous areas.

\subsection{The contradiction and relationship between power system planning construction and mountainous areas urbanization are simply processed by local governments}

Although the urbanization of mountainous areas is an innovative measure in Yunnan, it is also a new thing, in which the experience and lessons of the urban planning of other places can be introduced.

\section{Strategies for optimizing the power system planning in the urbanization of Yunnan's mountainous areas}

\subsection{Power supply enterprises should strengthen the communication with the government}

10 types of mountainous land development are promoted in the urbanization of Yunnan's mountainous areas, in which infrastructure projects represented by Kunming Changshui International Airport and Lijiang Railway Station, the industrial park projects represented by Yiliang and Pu'er industrial parks, the cultural and tourism projects represented by film and television, sports, and resorts in Tengchong and Bannan, the port construction projects represented by Mohan and Runli, the educational development projects represented by Chuxiong Vocational Education Park and Maitreya Middle School, the geological disaster prevention and control projects represented by the peak clipping, valley filling, stone carrying and land constructing projects in Lvchun County, the modern agriculture sightseeing projects represented by the mountain planting, breeding, and forestry in Malong county's modern agricultural park, and Wuhua District's Xizhu Ecological Experimental Zone, and energy projects represented by the hydropower, wind and large water conservancy facilities are involved. Power supply enterprises should actively strengthen the communication with local government and promote an effective connection between power system planning and local urban and rural planning, so that the coordinated development of power grid and local economy is enhanced.

\subsection{Including power system planning into the overall planning in the urbanization of Yunnan's mountainous areas}

Pilot projects have been actively, reliably developed in Yunnan's mountainous areas. The implementation plans of all areas have been approved step by step. According to the idea of starting, improving and storing projects, the pilot projects of constructing urban areas in mountains have been implemented in a planned way. In April 2012, spot-surveys were conducted by the Office of Land and Resources of Yunnan Province in the pilot projects of 10 areas; the comprehensive development and utilization project of low mountains and hills in Qujing had been started; field leveling work had been made on the experimental units in Honghe Luxi, etc. The power system planning work in all areas has been implemented at full stretch. In the process of power system planning, the traditional power system planning ideas should be broken, and the location and requirements of the power system planning should be synchronously considered from the conceptual planning. To dynamically integrate power system planning with mountainous areas urbanization planning, electric power enterprise must closely cooperate with local government in the process of making 
overall planning and also actively participate in the formulation of the general planning of urban and rural areas. The electric power special preparation process should be led by the government and guided by power supply enterprises, so that the achievements in this preparation are much easier to be implemented into the overall planning and detailed regulatory planning. The comprehensive connection and fusion between power system planning and the urbanization of mountainous areas will be finally realized once power supply enterprises participate in the overall planning and preparation.

\subsection{Making excellent load forecasting on power system planning based on the urbanization planning of mountainous areas}

Power load forecasting is the foundation of the power system planning, and accurate power load forecasting is the key to make successful power system planning. In the process of power system planning, the power load data in different areas must be collected, and power load must be forecasted using different methods. In the power system planning, the construction and development trend of urban road network and residential quarters must seriously be taken into consideration, and the construction scale and electro-characteristics of the industrial parks must be analyzed. Through rigorously analyzing the national economic development level, the flowing and growth trend of the stream of people and logistics is analyzed, and also the construction scale and progress of power system are reasonably planed.

\subsection{The power distribution network planning target positioning is necessarily based on the reality and can be in appropriate advance}

The power distribution network planning target positioning in the urbanization process of Yunnan's mountainous areas is necessarily based on the reality and insists on the sustainable development strategy. To determine the distribution network planning target positioning, the actual conditions of local areas must be combined, the investment, construction and transformation time, and quality problems must be considered as a whole. If the target is high, the quality is necessarily high, but the investment increase and construction prolonging are required, so that the effect of "the loss outweighs the gain" is obvious to all under the limit of the current investments; if the target is low, the investment decrease and construction shortening will be caused, but the quality is low, so that the problem of the quality not in line with the development and change needs occurs, and the problem of reconstruction and retransformation is necessarily to be solved when the construction and transformation are just completed. A balance among investment, time and quality can be found only if the target is rational, so that the quality is assured, but also the progress of the construction and transformation can be quickened under the limit of investment.

\section{Conclusion}

It is necessary to insist on the sustainable development strategy and co-construct a resource conservation-minded society. Planning conservation is the greatest saving, and therefore, the power grid enterprises in Yunnan should constantly optimize and improve the power grid structure and make the circuits closely combined with the transport planning according to the urbanization planning of mountainous areas and the requirements of resource conservation-minded society, so that the general plans for land can be easier. At present, the construction funds of the power supply enterprises are limited, and the products with good performance can be selected for outdoor equipment and those reliable manufacturers examined by practice are better chosen. The manufacturers should have ability in continuous operations and provide effective warranties for the future maintenance and upgrading of 
products. The use of new products is better seriously compared and analyzed from the perspective of technology and economy.

\section{Acknowledgement}

This paper aided financially by the Advantageous, Special, and Key Subject of the "Twelfth Five-year" Plan of Yunnan Province, "Applied Economics".

\section{References}

[1] Baidu's Encyclopedia. Cities and Towns in Mountains.

http://baike.baidu.com/view/6921391.htm.

[2] The urbanization rate of Yunnan Province is $35.20 \%, 10$ years Far Away from the National Level. http://yn.yunnan.cn/ html/2011-05/17/content_1615331.htm.

[3] Guibin WU. Discussion on the Power Grid Planning and the Coordination and Connection of the Overall Urban Planning [J]. Guangdong Science and Technology, 2011.11.
[4] Haibo ZHAO, Xingping JIA. Analysis on How to Make an Effective Connection between Power Grid Planning and Urban-rural Planning [J]. Journal of Information Science and Technology, 2012.29.

[5] Guangde HUANG. Analysis on the Re-planning of the Power Distribution Network in Mountainous Counties [J]. Guangdong Science and Technology, 2012.17.

[6] Qiang ZENG, Chunhua HU. Discussion on the Power Grid Planning in the Hilly Areas of South Sichuan Province [J]. Power Supply and Consumption, 2006.05.

[7] Youde WU, Yuanchang WANG. Study on the Special Urban Development Paths in Frontier Minority Areas-Yunnan is Taken for Example [J]. Journal of Yunnan Normal University (Philosophy and Social Sciences Edition), 2010. 